\title{
Prediction of time to flowering in soybean with artificial neural network
}

Taratuhin O. ${ }^{1}$, Novikova L. ${ }^{1,2}$, Seferova I. ${ }^{2}$, Samsonova M. ${ }^{1}$, Kozlov K. ${ }^{1}{ }^{*}$

${ }^{1}$ Peter the Great St. Petersburg Polytechnic University, St. Petersburg, Russia

${ }^{2}$ N.I. Vavilov All-Russian Institute of Plant Genetic Resources (VIR), St. Petersburg, Russia

*e-mail:kozlov_kn@spbstu.ru

A number of days from emergence to flowering is an important trait in soybean that strongly depends on temperature and day length. We investigate this dependence using mathematical modeling with Artificial Neural Networks (ANN). Experimental data was obtained in 1999-2013 in Leningradskaia oblast (Pushkin) and Krasnodarskii krai for nine early maturing soybean accessions with low photosensitivity. We adapted ANN to predict time to flowering in this dataset in two steps. Firstly, we added scaling constants for network inputs, optimized high and low temperature thresholds and base day length. Training of 121 model parameters resulted in the MSE value of 0.026. Investigated accessions were characterized by decreased high temperature threshold in comparison to literature data $\left(23^{\circ} \mathrm{C}<30^{\circ} \mathrm{C}\right)$ and increased low temperature threshold $\left(12^{\circ} \mathrm{C}>5^{\circ} \mathrm{C}\right)$. The increase of day length from $12 \mathrm{~h}$ to $13 \mathrm{~h}$ confirmed the adaptation to a longer day. The average error was $\sim 2.4$ days. ANN has to be trained for each group of plants from the same accession. Secondly, we modified the network topology to include the nine input nodes that represent the membership of a plant to an accession group. Thus the same ANN is used for any plant. An average error for the second model was $\sim 3.1$ days. The average errors for ANN models from both steps are less than the error of 5.2 days of the previous model based on temperature thresholds. We applied the first model to the generated weather for different future greenhouse gas emission scenarios and predicted flowering time for nine accessions in changing climate for years 2019-2030. Mean time to flowering decreases from measured 39.21 days to 36.33 days predicted for 2030 significantly with Mann-Witney-Wilcoxon criterion $(P=0.0097<0.01)$ but may stay constant or fluctuate in several cases.

Acknowledgements: The work was supported by Federal Contract \#14.575.21.0136 at 26.09.2017, RFMEFI57517X0136. 